\title{
Toxic Leukoencephalopathy by Accidental Oral Ingestion of an Infant's Fentanyl Patch
}

\author{
Hyeji Lim, MD, Hyunjoo Lee, MD, Young-Mock Lee, MD \\ Department of Pediatrics, Yonsei University College of Medicine, Seoul, Korea
}

Received: October 12, 2020

Revised: October 27, 2020

Accepted: October 29, 2020

Corresponding author:

Young-Mock Lee, MD

Department of Pediatrics, Gangnam

Severance Hospital, Yonsei

University College of Medicine, 211

Eonju-ro, Gangnam-gu, Seoul

06273, Korea

Tel: +82-2-2019-3354

Fax: +82-2-3461-9473

E-mail:YMLEEMD@yuhs.ac
The misuse and abuse of fentanyl patches for chronic pain have been frequently reported. Such reports show side effects ranging from asymptomatic to death [1]. However, there are no reports on brain and neurological damages resulting from oral ingestion of such patches. Thus, we would like to report on this subject.

A 10-month-old normally developed boy was admitted to emergency room of another hospital. He took a nap at 3:00 PM; however, did not wake up 3 hours. His presenting complaint upon arrival was as follows: severe drowsiness with inappropriate response to pain equivalent to a score of 6 on the Glasgow Coma Scale (GCS). His pupils were fixed, and both lower extremities showed spasticity on neurologic examination. The initial laboratory results showed lactic acidosis. Following oxygen supply, hydration, and sodium bicarbonate infusion, he showed irritability and a weak response to pain. Nonetheless, there was no spontaneous eye opening. He underwent emergent brain magnetic resonance imaging (MRI) that showed diffuse brain damage at the both frontal lobe, para-sagittal area, basal ganglia, occipital, and cerebellum (Fig. 1A and B). He was referred to Gangnam Severance Hospital for further treatment.

The initial GCS score at Gangnam Severance Hospital was 8 (eye opening response, 2; best verbal response, 2; best motor response, 4). We found a $12 \mu \mathrm{g}$ fentanyl patch in his mouth during physical examination. After removing this patch, we administered naloxone for detoxification. An electroencephalogram (EEG) performed on the day 2 showed a diffusely slow and disorganized background, without epileptiform discharges. The GCS score increased to 10 (eye opening response, 4; best verbal response, 2; best motor response, 4). He had a seizure on the day 3 . His arms alternatively became rigid, which later advanced to generalized tonic-clonic movement. He had ophthalmic examination due to lack of eye contact, which showed intact optic nerve. The brain MRI did not show any interval change on the day 11 (Fig. 1C and D). He could not control his neck or crawl. Despite residual neurological and visual impairment, his general condition improved, and he was discharged from the hospital.

He received follow-up brain MRI and EEG after 2 months. The EEG showed no significant change. However, there were symmetrical cystic changes in the entire brain areas, mimicking the improved MRI results in hypoxic insults (Fig. 1E and F). His visual impairments improved enough for his eyes to follow objects, and his neurological deficit improved as well allowing him to control his neck or crawl.

After 8 months, the brain MRI showed no interval changes compared to the earlier brain

Copyright (C) 2021 Korean Child Neurology Society

This is an Open Access article distributed under the terms of the Creative Commons Attribution Non-Commercial License (http://creativecommons.org/licenses/by-nc/4.0/) which permits unrestricted non-commercial use, distribution, and reproduction in any medium, provided the original work is properly cited. 

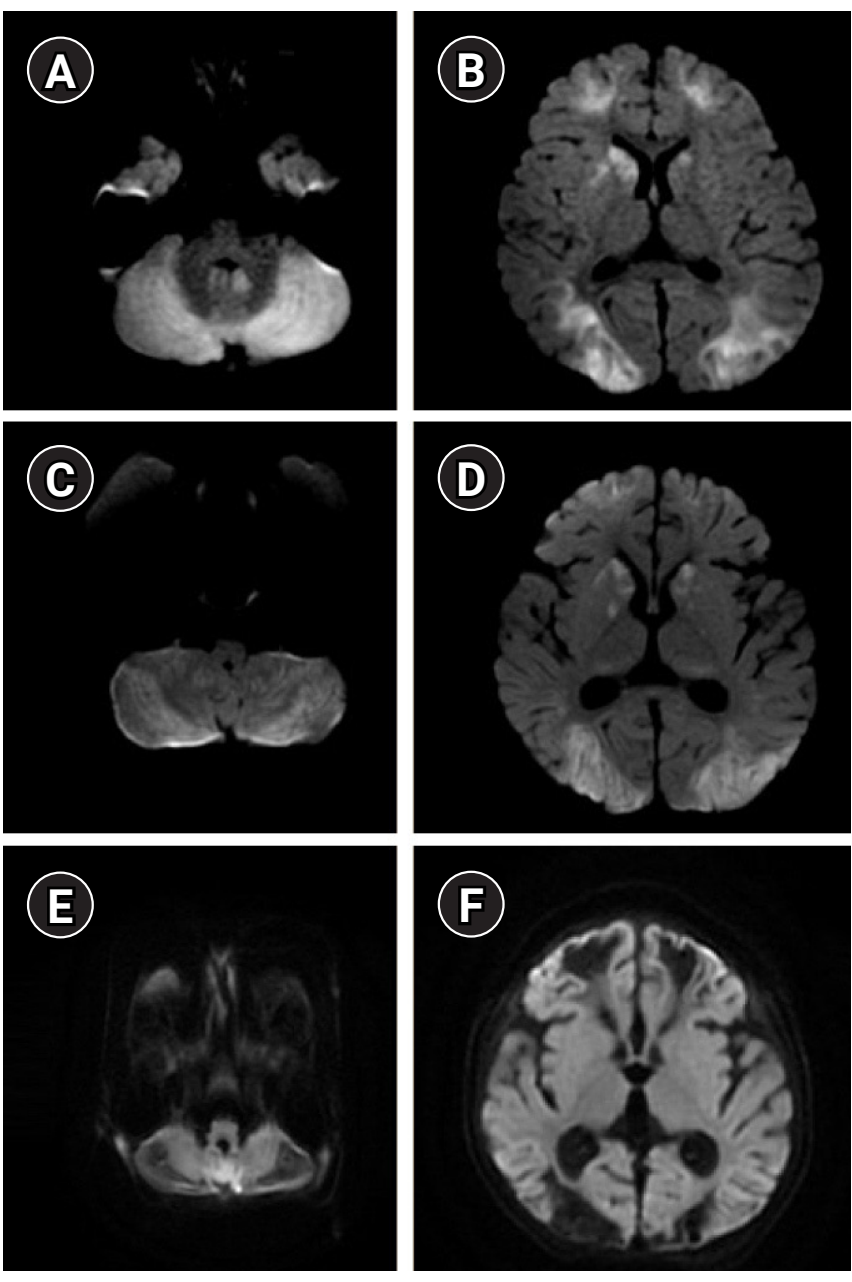

Fig. 1. Magnetic resonance imaging (MRI) diffusion-weighted imaging (DWI) images. (A, B) This is the initial DWI image of brain MRI. High signal intensity is shown in frontal, para-sagittal, occipital, and cerebellum, which means acute infarction. (C, D) DWI, which was implemented after 10 days, is not much different from $(A, B)$ and can identify toxic leukoencephalopathy pattern. $(E, F)$ Two months later, brain MRI showed a symmetrical cystic change in the overall brain, which is also identified as toxic injury insult.

MRI. Furthermore, frequent sharp waves were seen in his EEG but there were no clinical seizures. Nevertheless, he could toddle and utter about three words. His sight improved enough to recognize and hold objects. The brain damage persisted while his neurologi$\mathrm{cal}$ and visual deficiency was recovering.

In our case, the patient reported a neurologic change after orally ingesting a fentanyl patch. When a fentanyl patch is absorbed orally, the absorption of fentanyl is three times more than that absorbed through the stomach [2], which may be fatal to young children. A few numbers of accidental misuse of opiate pills revealed cerebellar damages and edema that can be attributed to opioid mu receptor toxicity $[3,4]$. However, unlike previous cases, our case il- lustrates overall brain damage from toxic leukoencephalopathy resulting in neurological and visual deficits. Such injury was caused by the toxic leukoencephalopathy from fentanyl intoxication.

This case of a 10-month-old illustrates that fentanyl intoxication may not only result in cerebellar damages and edema but also cause overall brain damage and neurological deficit in pediatric patients. We suggest that oral fentanyl intoxication should be considered as a differential diagnosis for children presenting with acute neurologic deficits showing diffuse brain damage due to toxic leukoencephalopathy on the brain MRI.

This study was approved by the Institutional Review Board of Yonsei University Health System (IRB, 3-2020-0358). Informed consent was waived by the board.

\section{Conflicts of interest}

No potential conflict of interest relevant to this article was reported.

\section{ORCID}

Hyeji Lim, https://orcid.org/0000-0001-7860-4574

Young-Mock Lee, https://orcid.org/0000-0002-5838-249X

\section{Author contribution}

Conceptualization: HL, HL, and YML. Data curation: HL, HL, and YML. Methodology: HL, HL, and YML. Visualization: HL, HL, and YML. Writing-original draft: HL. Writing-review \& editing: HL, HL, and YML.

\section{References}

1. Prosser JM, Jones BE, Nelson L. Complications of oral exposure to fentanyl transdermal delivery system patches. J Med Toxicol 2010;6:443-7.

2. Faust AC, Terpolilli R, Hughes DW. Management of an oral ingestion of transdermal fentanyl patches: a case report and literature review. Case Rep Med 2011;2011:495938.

3. Chen CH, Mullen AJ, Hofstede D, Rizvi T. Malignant cerebellar edema in three-year-old girl following accidental opioid ingestion and fentanyl administration. Neuroradiol J 2019;32:38691.

4. Foy L, Seeyave DM, Bradin SA. Toxic leukoencephalopathy due to transdermal fentanyl overdose. Pediatr Emerg Care 2011;27:854-6. 